\title{
Responsibility, Prevalence and major factors of infertility. A cross-sectional study in Karachi.
} Ayesha Khan, Neelam Barkat, Sidra Afzal \& Shehla.

Al-Hafeez Fertility And Medical Centre Civil Hospital Karachi

Dow University of Health Sciences

Corresponding Author:sidra.afzal@hotmail.com

\begin{abstract}
A cross-sectional study was conducted to target the infertile couples visiting to private and government hospitals in Karachi from 2012 to 2014. The aim of the study is to know the higher prevalence of infertility among the married couples and to determine the major factors causing infertility. Detailed evaluation had been made to assess each cause. Likewise, in females, we analyze menstrual history, parity, past surgical history, family history and body weight, years of marriage, complete hormonal profile and ultrasonic measurements. On the other hand, in males; we conduct complete analysis of semen, coital frequency, addiction to any substance and medical history. Overall 475 infertile couples were targeted in the study, in which 175 pairs were included, who full fills all the inclusion criteria, i.e. with complete medical investigations and excluded the remaining with incomplete reports in females and in males who denied semen evaluation. The included couples are divided into four groups in order to determine the responsibility of childlessness between the two, a) female, b) male, c) both and d) unexplained infertility. Our study concludes that in the Karachi the majority of females are responsible for unproductiveness up to $41.1 \%$ rather than the male up to $25 \%$, whereas $16.5 \%$ both the couples are involved in it and $17 \%$ has unexplained screened problems. Moreover, our study further explores the occurrence of primary infertility which is twice, i.e. $71.1 \%$ than secondary infertility, i.e. $22.8 \%$ in Karachi city. However, the major female factor is PCOS which is observed in more than half of the females in both primary and secondary incidents. While, lower sperm count, inefficient sperm motility and higher rate of abnormal sperm are the key male factors.
\end{abstract}

\section{Introduction}

Infertility is a disease or a condition of reproductive system that interferes with the ability to conceive. In a society like Pakistan with conventional and extensive families, there is a lot of anxiety on the couple to have a baby. The main role of women is still seen to be one of perpetuating the family name. Hence, the analysis of infertility can be a mug on relationship, self-image and sexuality. The occurrence of infertility has been noted to be greatly variable worldwide. Global estimation proposed that nearly 72.4 million of couples experiencing fertility problems (Gurunath; 2011). Whereas, about $25 \%$ of European and American couples experiencing reproductive disorder (Mohammad Reza safarinejadi; 2006).

In contrast, constantly an average rate of $10-15 \%$ has been quoted in develop countries (Garcia JE; 2008, Bhattachaya S; 2007 \& Alvarez NC; 2006). The incidence of infertility in Pakistan is quarterly noticed; in which majority suffers from secondary infertility as compared to primary infertility (Gurunath; 2011, \&Wood C; 1995). Infertility can be classified as primary when the women have never conceived and secondary when she has achieved pregnancy before, but facing failure in future trials.

The etiology of infertility may be from any partner or both. However, etiology cannot be found in average $17 \%$ of the couples (Kumar A; 2007). The reproductive method requires the contact of the female and male reproductive tracts, which will permit development and release of oocyte by the ovaries, production of sufficient spermatozoa by the testes, the normal transportation of the gametes to ampullary portion of the fallopian tubes where fertilization takes place and carrying of the embryo to the endometrial cavity for implantation and further development. Any aspect that affects the effects any of the routes can originates infertility.

Causes of male infertility include; problems of development of sperms like Azospermia, Oligzospermia, Teratozospermia and Asthenozospermia. Hindranceof the ejaculatory ducts, which can be an outcome of infection, can also cause infertility. Tobacco and alcohol intake have been found to be risk factors for infertility in both partners.

Causes of female infertility include; ovulatory disorders, tubal blockage, uteroperitoneal factors like uterine synaechia, endometriosis and uterine fibroid. The part of sexually transmitted infection in causing infertility is well-known.

Male factors can be evaluated by seminal fluid analysis. Ultrasonography can be used to evaluate the scrotal volume. Testicular biopsy has a minimum role in the estimation of male factor infertility.

Treatment options depend on the age of the patient, cause, financial consideration, expertise and facilities available. The key treatment for male factor is tubal and male ejaculatory duct microsurgery, intrauterine insemination, in-vitro fertilization and intra-cytoplasmic sperm injection. However, the last choice is very luxurious and not readily available in developing countries. Adoption remains a possible option for some couples (Garcia JE; 2008). The aim of this study is to determine the responsibility, prevalence and major causes of infertility in our environment.

The study consisted of 475 couples who visited fertility clinics of centrally localized one private and a government setup, i.e. AlHafeez fertility \& medical centre and civil hospital from 2012 to 2014. These two years of study period aim to evaluate prevalence of infertility among the couples of Karachi who visited in centers, complaining unproductiveness. Additionally, this study further leads to establishment of major causes that are creating a hurdle in conceiving.
}

We included 175 patients who completed and followed total evaluation format, others were excluded. At first visit clinic's routine management protocols were implemented, referral status form was filled up by the couple .which included both partner's basic medical history .Data included age, prior hospitalizations or chronic illnesses, usage of any medications, duration of trial for conception, any addiction habits, any previous surgery. For women, dysmenorrhea, menstrual regularity, pelvic inflammatory disease and dyspareunia. After that, couples were referred for further evaluations initially with a hormonal analysis in females (luteinizing hormone ,follicle-stimulating hormone, thyroidstimulating hormone, prolactin, hormone, DHEAS and estradiol ) and for men complete semen analysis was performed, blood test and ultrasounds were performed in case of disturbed semen parameter. After all these reports collection if it was 
necessary females were asked to went through tubal potency test, i.e. hysterosalpingography (Fig : 1e). HSG is not preferred in cases of known male factor, severe endometriosis or pelvic factors (Fig : 1a-1d)

Infertility causes were defined according to following criteria. When a menstrual cycle is of more than 35 days it will be classified as anovulatory/ oligo infertility. Existence of minimum two of Rotterdam criteria (Bhattacharya S; 2007). Within this category will be defined as polycystic ovary syndrome. Annovulation and polycystic ovary syndrome was investigated initially by ultrasound and other factors associated with these complication was investigated by day 2 hormonal profile (table 1 ); by FSH and LH concentration (Alvarez NC; 2006).
Male Factor was evaluated by routine semen parameter (Fig: 2a$2 \mathrm{c}$ ), $<50 \%$ sperm motility (asthenozoospermia); low sperm count of $<20 \times 106$;(sperm cells/ml (oligozoospermia); and/or abnormal morphology of $<14 \%$ according to the criteria of Kruger et al or $40 \%$ according to World Health Organization criteria (United Nations Summit; 2010), (Araoye OM; 2003). (teratozoospermia).all the factors when reported in normal limits the condition was defined as Unexplained infertility. After completing all the required evaluation we assessed the data carefully to obtain the ratio of primary and secondary infertility in Karachi, and factors which are mostly responsible for this problem and major causes in both partners leading towards infertility.
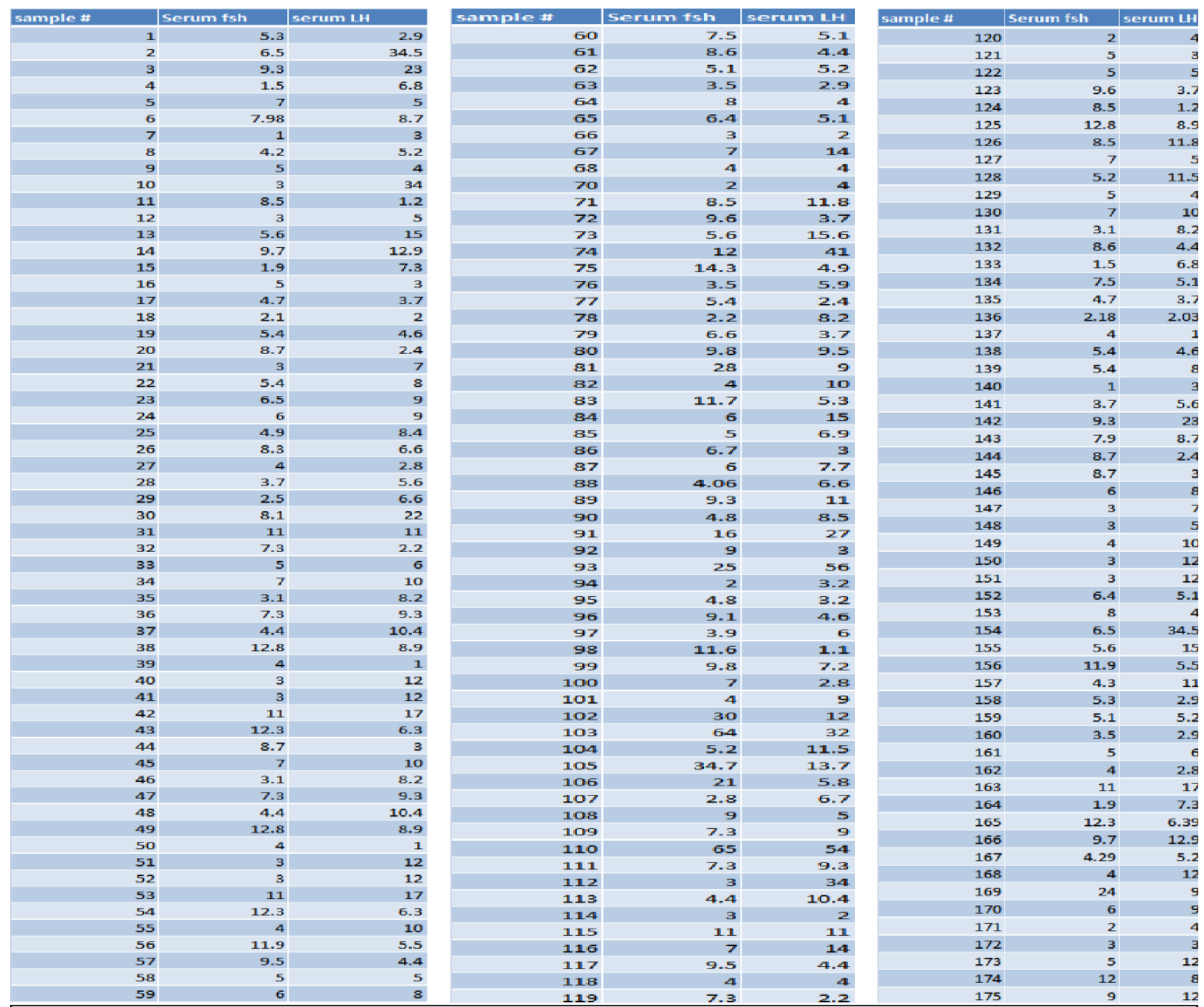

Table 1. Showing ratio of Serum FSH and serum LH (menstrual cycle day 2) of all the included infertile females of Karachi. 

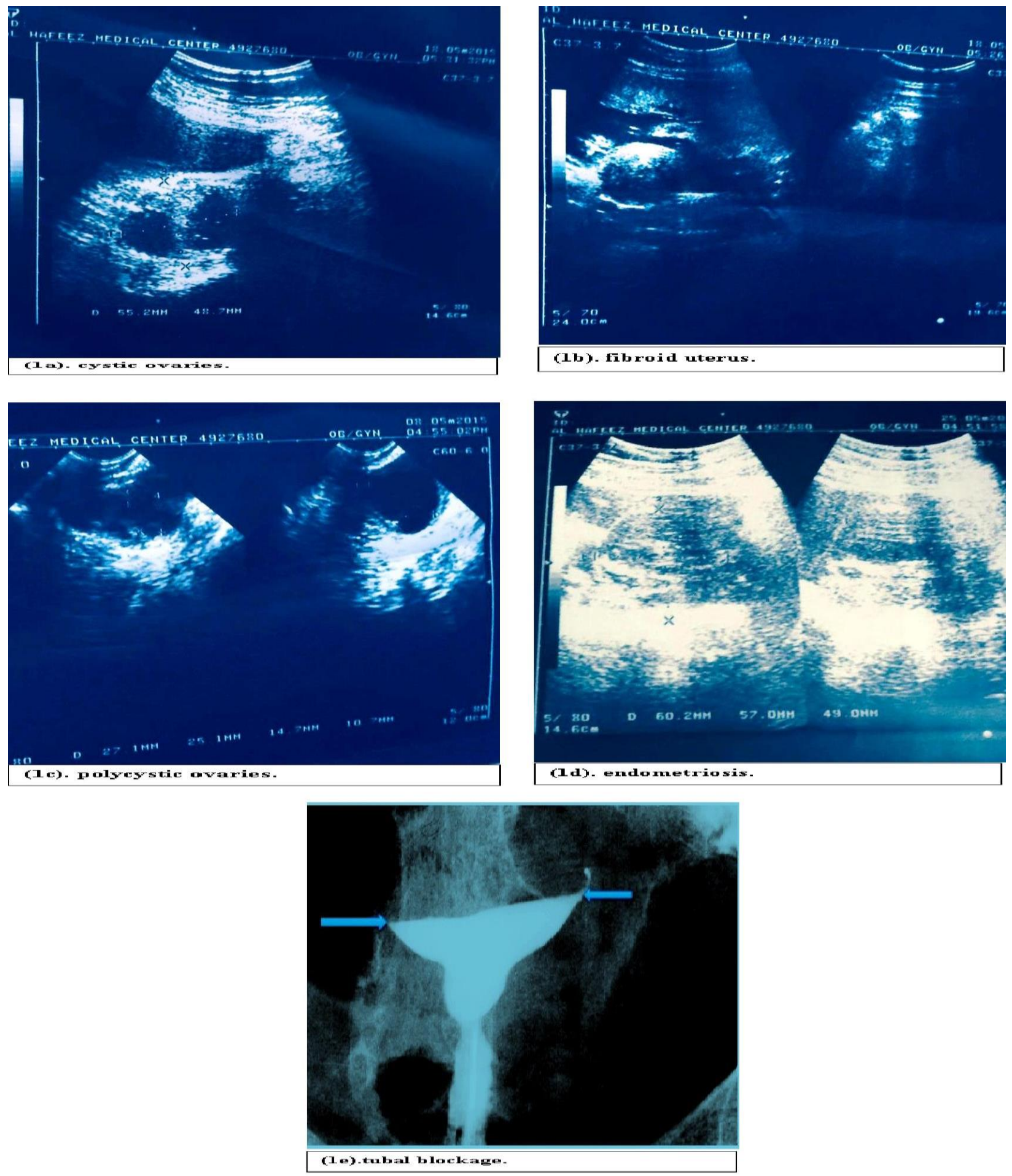

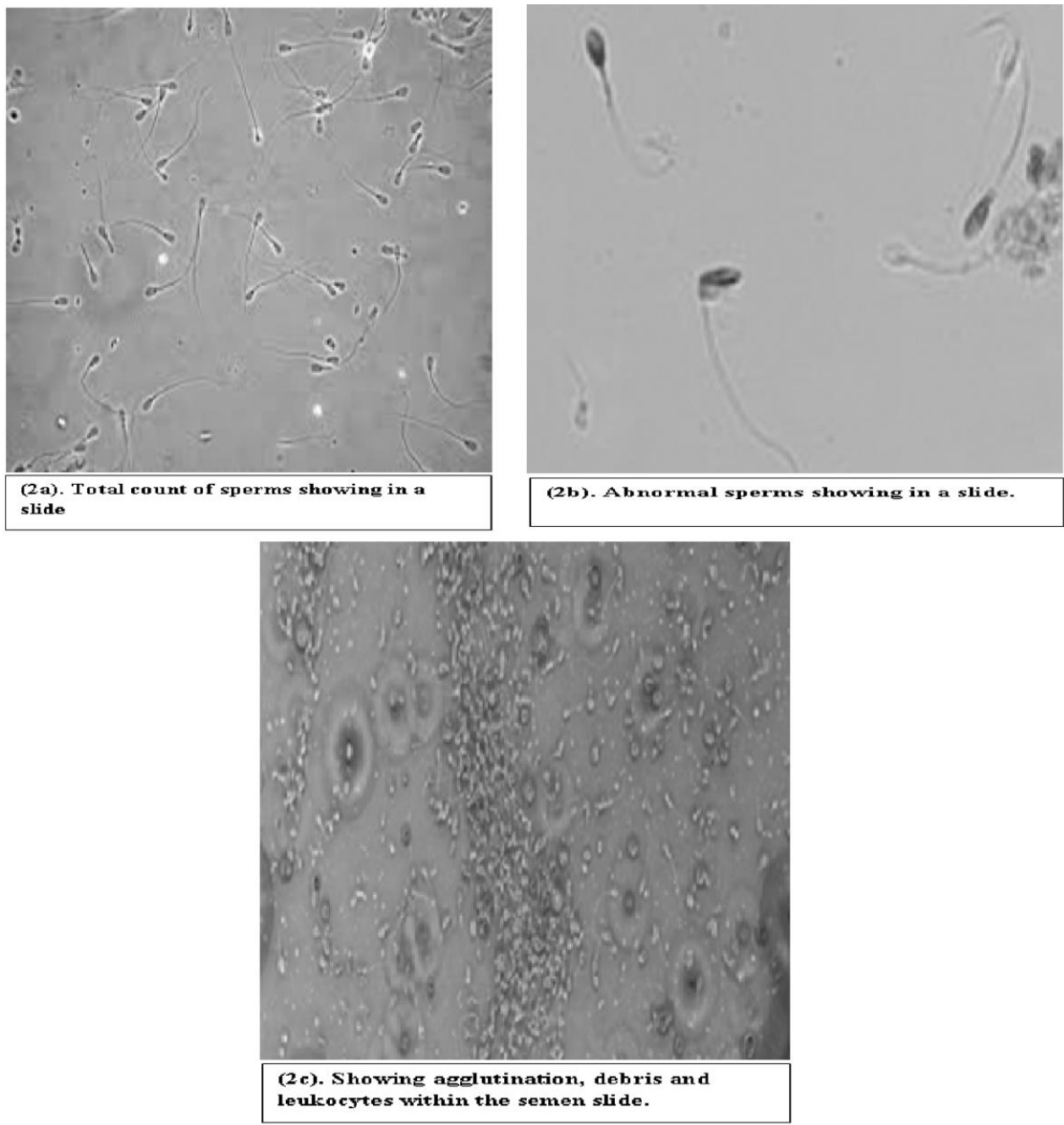

\section{Results}

The graph 3(a) describing the incidence of higher primary infertility (couples who had never conceived even after trying for 1 year without using any protection) that is thrice the occurrence of secondary infertility (couples who had conceived once but now facing failure in further trials)

The graph 3(b) demonstrating the higher contribution of females towards infertility of a couple $(41.1 \%)$ which is nearly half in total as compared to males which are quarterly responsible in this problem ( $25 \%$ ). While, both partners simultaneously contributing for infertility were reported in almost $16.5 \%$ of cases. Whereas, in $17 \%$ of couples the causes were remain unknown even after complete workup.

The graph 3(c) showing different pelvic factors of females that causing hindrance in achieving the pregnancy. Polycystic ovary syndrome was found to be the highest pelvic contributor toward complication in achieving pregnancy as it enhances the complications in ovulation and menstrual periods, second most pelvic disorder was blockage of fallopian tubes that develops a barrier between female oocyte and male spermatozoa that leads to a failed fertilization.

The graph 3(d) defining the major male factors that causing infertility among the couples; teratozoospermia i.e. abnormal morphology of sperms which was the highest factor that unable to fertilize female egg. However, azzospermic was the second most condition in which sperm count was nil that triggered infertility and oligospermia; low concentration of sperms was also noted, with asthenospermia; low motility of sperms which also created hurdle in fertilization. 


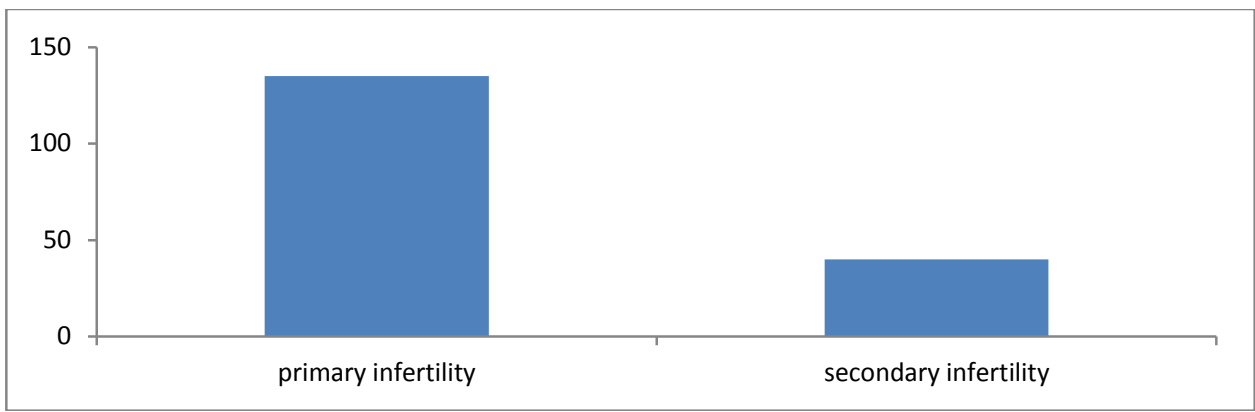

3(a).Table showing the higher ratio of primary infertility $71.1 \%$ as compared to secondary infertility $22.8 \%$ in Karachi city.

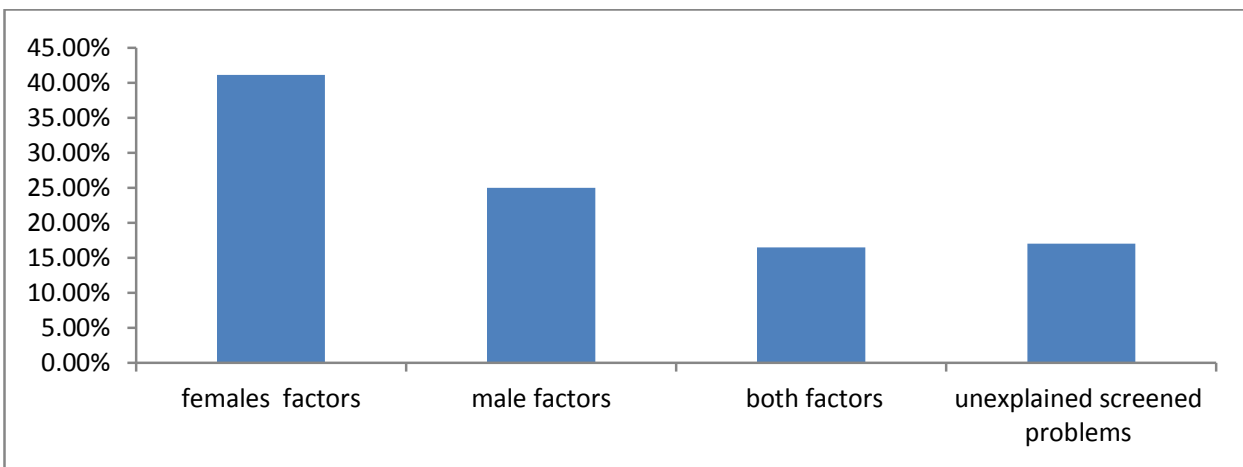

3(b). Table showing that in Karachi majority of females are responsible for unproductiveness up to $41.1 \%$ rather than male up to $25 \%$, whereas $16.5 \%$ both the couples involved in it and $17 \%$ of unexplained screened problems.

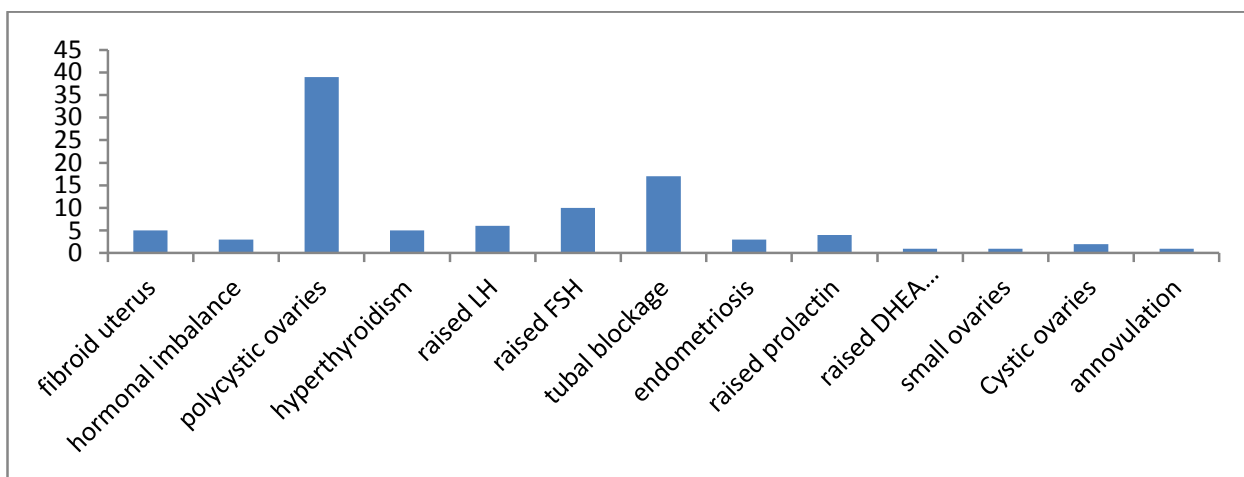

3(c).Table showing that the major female factor is PCOS which observed in more than half of females in both primary and secondary infertility incidents.

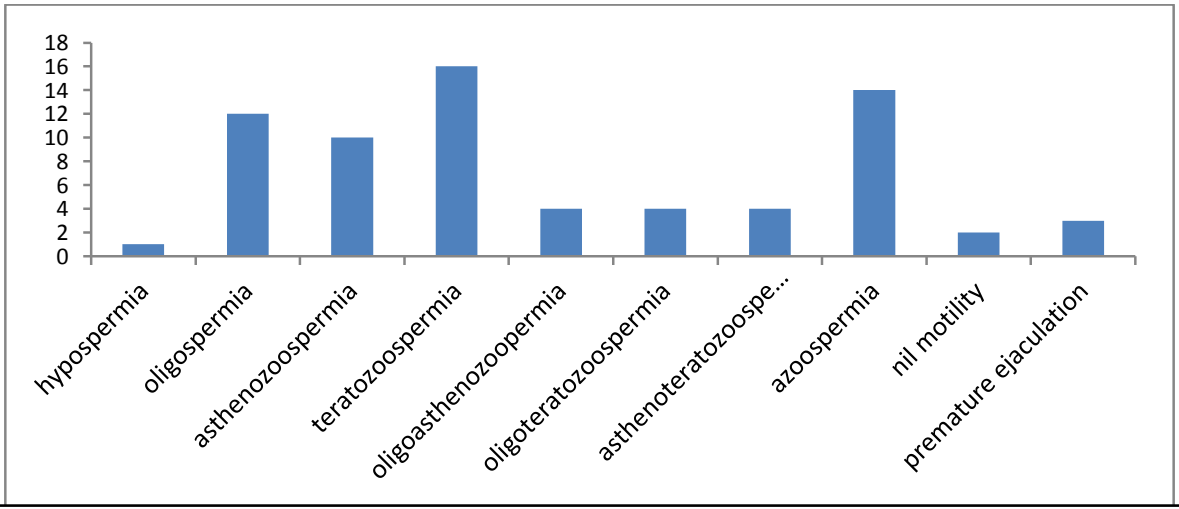

3(d). Table showing that the low sperm count, inefficient sperm motility and higher rate of abnormal sperm are the key male factors contributing to infertility. 


\section{Discussion}

The women with infertility are socially burdened and irrespective of the fact that which one among the two is responsible for this infertility have to bear all these brunt (Neelofar Sami; 2006). Similar study conducted in India also reported the same thing (Riessman CK; 2000). It's a common practice in traditional South Asian countries that a woman gains security and prestige in her in laws only after she proves her fertility and gives them a child (Unisa S; 1999). Within the social setup of Pakistan, there is a huge pressure on the newly wedded couple for bearing a child. There is a high prevalence of childlessness in Pakistan (Tahir et al., 2004).

Infertility generally defines any form of abridged fertility with lengthened time of wanted conception. In a review (Gnoth et al., 2005) it has been reported that with intercourse in the first six cycles most of the pregnancies occurs in the productive phase $(80 \%)$. After this time period, serious infertility must be implicit in every second couple (10\%), after 12 unsuccessful trials, nonconception rates in coming 36 months among them will attain almost 55\%. After (48 months), in approximately 5\% of the couples there is definite infertility with zero percent of chances to get pregnant in future.

According to World Health Organization (WHO) estimation there are worldwide 50 to 80 million couples who are not fertile (Montoya JM; 2002).

Many populations were studied to determine the Causes and prevalence of infertility. In different populations it has different rates of prevalence (Wood C; 1995).

Pakistan is one of the most populated countries in the world with the population escalation rate of around $2 \%$, it is also facing high ratio of infertility $(21.9 \%) ; 3.5 \%$ primary and $18.4 \%$ secondary (Tahir et al., 2004). In this study of 475 couples of Karachi city, 175 were included who had tried to conceive and had complete medical investigations, it was found that about $71.1 \%$ were facing primary infertility whereas $22.8 \%$ were facing secondary infertility which indicates the prevalence of primary infertility more than three times as compare to secondary infertility which is totally opposite the country wide estimation (Tahir et al., 2004). According to WHO both of the partners are somewhat equally responsible for their infertility like male partner is responsible in about $20-25 \%$ of cases same as with females (WHO Standards; 2010). For the first time our data provided indication of female to be more responsible than male for the unproductiveness of a couple in Karachi i.e. about $41.1 \%$ whereas in $25 \%$ of the couple males were responsible for their childlessness, about $16.5 \%$ both of the couple was responsible and in about $17 \%$ of the couple the problem was unexplained.

Another purpose of the study was to evaluate the factors contributing towards infertility, different uterine anomalies in females leads them towards infertility, according to our findings in Karachi population polycystic ovaries is the biggest reason of infertility in females of reproductive age as it is the condition that leads to many other abnormalities like higher testosterone and mid follicular LH which causes disturbance in menstrual and ovulation cycle. A Study indicates that family history has a profound effect in the development of polycystic ovarian syndrome (Sadaf A; 2013). Another study reveals the problems associated with polycystic syndrome are due to improper dietary habits that are making Karachi females more prone to develop the disorder (Shamoon N; 2015). Its prevalence is higher in ovulatory women as compared to normal population, it suggest that $\mathrm{PCO}$ by its effect of hyperandrogenaemia contribute to infertility (E. Kousta; 1999). Second largest factor was found to be tubal blockage; it is the condition in which a major blockage inside the fallopian tubes causing the failure of sperm to reach oocyte and fertilize it. Whereas other reported factors were endometriosis, fibroid uterus and hormonal fluctuations. Whereas, in males disturbed semen parameters were observed. The abnormality that was observed in majority of the males was teratozoospermia sperms that are abnormal in morphology fails to fertilize oocyte. Secondly, azzospermia that is absence of sperm in ejaculate, which is the concern cause of infertility. Thirdly, oligospermia, i.e. low sperm count and lastly asthenospermia i.e. Low motility of sperms was the contributing factors.

Some limitations were encountered during the study, face to face interview were required. However incorrect information was provided by some interviewees like in women it was age and in male's addiction to some substance as well as some male partners denied for semen analysis thinking that they are perfectly fine. Finally $36.8 \%$ of couples were evaluated on the basis of completing the inclusion criteria.

\section{Conclusion}

This study concludes that the prevalence of primary infertility is much higher in Karachi city as compare to secondary infertility that is contrary to national reports. Another conclusion derived from the study was that, female partners are more responsible for infertility than male partners. Females are mostly victim of polycystic ovaries that is observed in both type of infertility. While, in male partner abnormal sperms and deficiency of sperms are the key factors leading to infertility. Further studies in other cities are recommended to better estimate the prevalence of infertility in Pakistan.

\section{Conflict of Interest}

We have read and understood IJEHSR policy on declaration of interests and declare that we have no conflict of interests.

\section{Acknowledgment}

This research was supported by Al-Hafeez fertility and medical centre, civil hospital Karachi and DUHS. We thank our colleagues from Al-Hafeez fertility and medical centre who provided insight and expertise that greatly assisted the research. We are also grateful to our mentors Dr Sadaf Ahmed and Sir Shamoon Noushad for their immense guidance.

\section{References}

- Alvarez NC. (2006).Infertility: the magnitude of the problem Rev Enfem, 29(5), 59-62.

- Araoye OM. (2003).Epidemiology of Infertility: Social Problems of the Infertile Couples. West Afr J Med, 22(2), 190196.

- Bhattacharya S. (2007). Infertility. In: Edmonds DK (Ed). Dewhurst's Textbook of Obstetrics and Gynaecology. Blackwell Publishing, 7, 440-460.

- E. Kousta1,3, D.M. White2, E. Cela2, M.I. McCarthy1 and S. Franks2.(1999 Nov).The prevalence of polycystic ovaries in women with infertility.Human reproduction, 14(11), 2720-23.

- Garcia JE, Nelson LM. Infertility (Assessed March 21 2008).

- Gnoth, c., godehardt, e., frank-herrmann, p., friol, k., tigges, j. And freundl, g., 2005. Definition and prevalence of subfertility and infertility. Hum reprod., 20:1144-1147.

- Kumar A, Ghadir S, Eskondari N, Decherney AH. (2007). Reproductive Endocrinology and Infertility. In: Decherney AH, Nathan L, Goodwin TM, Laufer N (Eds). Current Diagnosis and Treatment in Obstetrics \&Gynaecology. McGraw Hill medical publishing Division, 10, 917-925.

- Mohammad Reza Safarinejadi, (2006).Infertility among couples in a population-based study in Iran: prevalence ans associated risk factors. International Journal of andrology, ISN0105-6263.

- Montoya JM, Bernal A, Borrero C. (2002).Diagnostics in assisted human reproduction. Reprod Biomed Online, 5, 198210.

- New 2010 WHO Standards (5th Edition) for the Evaluation of Human Semen MahmoodMorshedi Ph.D., HCLD(ABB) 
- Neelofar Sami, TazeenSaeed Ali. . (Jan 2006). Psycho-Social Consequences of Secondary Infertility in Karachi.Department of Community Health Sciences, Aga Khan University, Karachi.

- S. Gurunath, Z. Pandian, Richard A. Anderson , and Siladitya Bhattacharya.(2011).Defining infertility - a systematic review of prevalence studies. Human Reproduction update, vol 17, issue 5,pp 575-588.

- Riessman CK. (2000). Stigma and everyday resistance practices: Childless women in South India. Gender and society, 14, 111-135.

- Sadaf A, Shamoon N, Neelam B, Sidra A, Zeeshan F. (2013). Impact of Family History in Development of PCOS. International Journal of Health and Education, 2, 62-72.

- Shamoon N, Sidra A, Neelam B, Sadaf A\&Nighat S. (2015). Atrocious dietary habits leading females of Karachi towards polycystic ovary syndrome. International Journal of Endorsing Health Science Research, Volume 3 Issue 1, 14-16Wood C, Dawson K. Assisted fertilization. In: Sachs B, Beard R, Papiernik E, Russell C, Editors.(1995). Reproductive health care for women and babies. 1st ed. Oxford: Oxford University Press, p. 322-45.

- Tahir, f., shahab, m., afzal, m., subhan, f., sultan, s., kazi, b. M. And dil, a.s., 2004. Male reproductive health: an important segment towards improving reproductive health of a couple. In: population research and policy development in pakistan (eds. M.a. Chaudhry and m. Desousa), pp. 227-248. Population association of pakistan, islamabad, pakistan.

- United Nations Summit on Millenium Development Goals. New York September 2010.

- Unisa S. (1999). Childlessness in Andhra pardesh, India: treatment-seeking and consequences. Reproductive Health Matters, 7, 54-64

- Wood C, Dawson K. (1995). Assisted fertilization.In: Sachs B, Beard R, Papiernik E, Russell C, Editors.Reproductive health care for women and babies.1st ed. Oxford: Oxford University Press, p. 322-45. 